\title{
63. THE EFFECT OF THE ELLIPTICITY OF JUPITER'S ORBIT ON THE CAPTURE OF COMETS TO SHORT-PERIOD ORBITS
}

\author{
E. EVERHART \\ Dept. of Physics, University of Denver, Denver, Colo., U.S.A.
}

\begin{abstract}
Single, random, close encounters of long-period comets with Jupiter are studied. In contrast to earlier work, Jupiter's orbit is taken to be elliptical, but this has no effect on the rate of capture into short-period orbits and neither does it influence the distribution of longitudes of perihelion.
\end{abstract}

Short-period comets are not distributed uniformly with regard to their longitudes of perihelion $\tilde{\omega}$. Considerably more of these comets have perihelia on one side of the solar system than the other. The distribution is shown in Figure 1a for the 98 known short-period comets. The longitude of perihelion of Jupiter is marked by the arrow on the figure and, evidently, a preponderance of the short-period comets more or less line up their orbits with Jupiter's. Figure $1 \mathrm{~b}$ shows that the same situation holds for a smaller group of 68 comets, these being all those of perihelion distance less than 2 $\mathrm{AU}$ and period less than $22 \mathrm{yr}$.
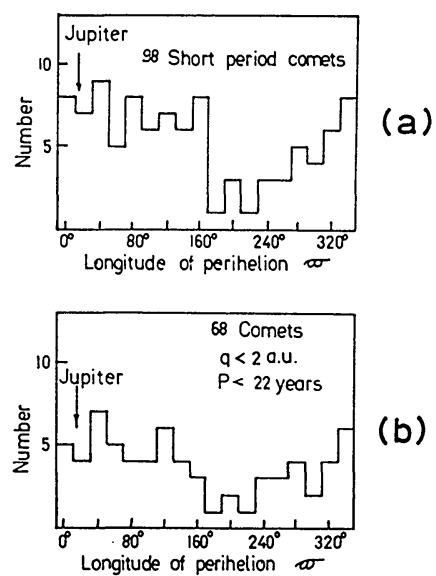

(b)

Fig. 1. Distribution of short-period comets with regard to longitude of perihelion $\tilde{\omega}$.

The capture hypothesis is that short-period comets originate when long-period comets pass near Jupiter and lose heliocentric energy to Jupiter during the encounter. (It is possible in principle for other planets to have a similar effect in capturing a comet, but the effects of Jupiter vastly predominate.) The first quantitative discussion of the capture hypothesis was that by Newton (1893). More recently, the present author (Everhart, 1969) has tested this hypothesis extensively in a Monte-Carlo calculation, taking $10^{9}$ hypothetical random parabolic comets interacting with the solar system, finding how many of these would be captured to orbits of short period, and obtaining 
the distributions to be expected among the captured comets in such quantities as period and inclination. This recent study, as well as that by Newton, assumed circular orbits for Jupiter and the other planets, a simplification that appeared to be reasonable for treating a rather complicated problem. However, because of symmetry a circular orbit for Jupiter could not possibly account for a nonuniform distribution of longitude of perihelion. Indeed, it was thought possible that taking Jupiter's orbit as elliptical would make changes in some of the other results as well.

Accordingly, the entire study of capture of comets by Jupiter is here repeated using an ellipse for Jupiter's orbit. The new calculation is somewhat more extensive and complicated than before, partly because of the transcendental equations that arise on finding the collision sites in the elliptical case, and partly because of the new requirement that Jupiter's mean anomaly must have a random value at the time each hypothetical comet comes to perihelion. The new result can be stated very simply: taking Jupiter's orbit as elliptical rather than circular makes no discernible difference in the results from the previous work. For example, the distribution of periods of the hypothetical short-period comets after capture has the same shape as before, and the scale factor is unchanged. The same result holds for the distribution of inclinations after capture and for all the other distributions plotted by Everhart (1969). The previous studies with a circular orbit for Jupiter gave distributions that did not agree with those of known short-period comets, and this lack of agreement is not changed in any way by taking Jupiter's orbit to be elliptical.

These results discussed so far have been averaged over Jupiter's orbit. One might inquire whether Jupiter captures a little more effectively near perihelion than near aphelion, or whether the opposite is true. Such an effect would show if the captured hypothetical comets were sorted according to where the encounter with Jupiter took place.

The results of such sorting are seen in Figure 2a, which shows the number distri-
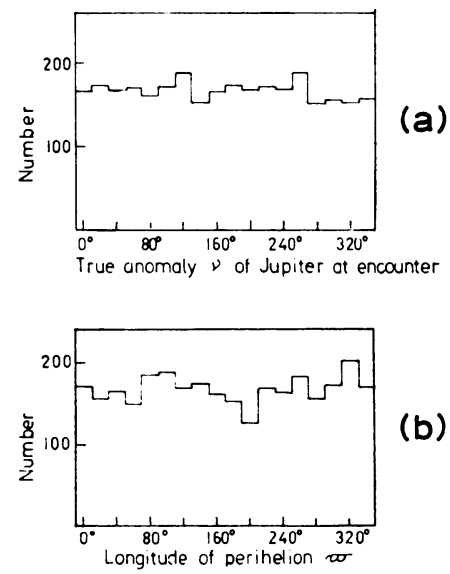

(b)

Fig. 2. Distribution of 3000 hypothetical comets captured to periods less than $22 \mathrm{yr}$ with respect (a) to true anomaly $v$ of Jupiter at the time of close encounter, and (b) to the longitude of perihelion $\tilde{\omega}$ of the captured hypothetical comets. 
bution of hypothetical comets captured to periods less than $22 \mathrm{yr}$ plotted versus the true anomaly of Jupiter at the time of the close encounter; and again in Figure $2 b$, which shows the number distribution plotted versus the longitudes of perihelion of the comets after being captured to periods of less than $22 \mathrm{yr}$. Both these histograms are uniform within statistical fluctuations. In the search for a possible effect many other distributions were examined: for example, those of inclination and period for comets encountering Jupiter in specified parts of its orbit. The same negative result is found in all cases. The eccentricity of Jupiter's orbit has no noticeable effect on the ability to capture comets to a short-period orbit in a single encounter.

Accepting this result then, we suggest three other possibilities that might account for the nonuniform distribution of $\tilde{\omega}$ seen in Figure 1.

(1) An observational selection effect. Although there has been no thorough study of observational selection of short-period comets, a somewhat similar asymmetry in a property of long-period comets can be so explained: it turns out that considerably more long-period comets have an argument of perihelion $\omega$ between 0 and $\pi$ than between $\pi$ and $2 \pi$. In a quantitative study (Everhart, 1967), this was shown to be an observational selection effect traceable to the fact that there have been more observers finding comets from northern observatories than from southern.

(2) The effect may be nonexistent. One may consider that the distribution of longitudes of perihelion is intrinsically uniform, but that statistical fluctuations in a small sample cause an apparent asymmetry. The well known chi-square test finds that there is a $5 \%$ chance of such an asymmetry arising statistically as in Figure 1a and a $25 \%$ chance as in Figure 1b. Unfortunately, one cannot repeat the plot with new data for 100 different short-period comets!

(3) The asymmetries seen in Figure 1 might conceivably be the result of Jupiter's orbital eccentricity if there were a small but cumulative effect in repeated encounters. This possibility has yet to be tested.

\section{References}

Everhart, E.: 1967, Astron. J. 72, 1002, Fig. 5.

Everhart, E.: 1969, Astron. J. 74, 735.

Newton, H. A.: 1893, Mem. Natl. Acad. Sci. Washington 6, 7.

\section{Discussion}

E. I. Kazimirchak-Polonskaya: Which perturbations did you take into account?

E. Everhart: Those by the Sun when the comet was in Jupiter's sphere of influence and those by Jupiter when it was in the Sun's sphere of influence. The calculation used a hyperbolic encounter formula that agrees within a few percent with exact numerical calculations of such encounters.

L. Kresák: It appears surprising that you do not find any asymmetry in the expected distribution of perihelion longitudes. Because of the law of areas, there is both a greater probability of encounter and a slower mean relative motion near Jupiter's aphelion.

$E$. Everhart: The formula I derived for the change in energy during a close encounter depends on the impact parameter, the relative velocities, and the angle between certain vectors. It is not diffcult to see that, to the first order. capture at Jupiter's perihelion and capture at aphelion should be about the same. 
G. Sitarski: You showed earlier that the probability of capture of a comet by Jupiter is very small: for example, one observable periodic comet in some thousand years. Why do you now present new investigations based on the assumption that periodic comets originate through capture?

E. Everhart: I did this merely to see whether the eccentricity of Jupiter's orbit would make any difference. I did not expect to find more captures in the elliptical case than in the circular case, but I did expect to find some positive effects and was surprised not to see anything. 